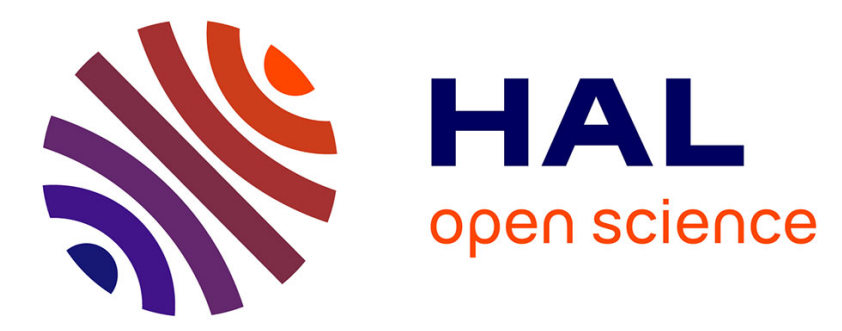

\title{
COMPUTATION OF MEAN FIELD EQUILIBRIA IN ECONOMICS
}

\author{
Aimé Lachapelle, Julien Salomon, Gabriel Turinici
}

\section{To cite this version:}

Aimé Lachapelle, Julien Salomon, Gabriel Turinici. COMPUTATION OF MEAN FIELD EQUILIBRIA IN ECONOMICS. M3AS, 2010, 20 (4), pp.567-588. 10.1142/S0218202510004349 . hal$00346214 \mathrm{v} 2$

\section{HAL Id: hal-00346214 \\ https://hal.science/hal-00346214v2}

Submitted on 9 Jun 2009

HAL is a multi-disciplinary open access archive for the deposit and dissemination of scientific research documents, whether they are published or not. The documents may come from teaching and research institutions in France or abroad, or from public or private research centers.
L'archive ouverte pluridisciplinaire HAL, est destinée au dépôt et à la diffusion de documents scientifiques de niveau recherche, publiés ou non, émanant des établissements d'enseignement et de recherche français ou étrangers, des laboratoires publics ou privés. 


\title{
Computation of mean field equilibria in economics DRAFT
}

\author{
Aimé Lachapelle, Julien Salomon and Gabriel Turinici \\ CEREMADE, UMR CNRS 7534, Université de Paris-Dauphine, \\ Place du Maréchal De Lattre De Tassigny, 75775 Paris cedex 16, France.
}

\begin{abstract}
Motivated by a mean field games stylized model for the choice of technologies (with externalities and economy of scale), we consider the associated optimization problem and prove an existence result. To complement the theoretical result, we introduce a monotonic algorithm to find the mean field equilibria. We close with some numerical results, including the multiplicity of equilibria describing the possibility of a technological transition.
\end{abstract}

keywords: Mean field games, dynamic equilibrium, externalities, monotonic algorithms, optimal control, non-convex optimization.

\section{Introduction}

The mean field games (hereafter MFG), introduced recently by Jean-Michel Lasry and PierreLouis Lions (Ref. [10, 11, 12]), appear to be well adapted to economic modeling.

While in standard game theory there are a finite number of players, MFG studies the behavior of a continuum of agents.

Over a finite time horizon $T$, each rational player minimizes a criterion:

$$
\mathbb{E}\left\{\int_{0}^{T} L\left(X_{t}, \alpha_{t}\right)+V[m]\left(X_{t}\right) d t\right\}
$$

over all eligible controls $\alpha_{t}$. The state of a generic player is $X(t)$ (also denoted $X_{t}$ ), which is a diffusion process governed by the controlled stochastic differential equation $d X_{t}=\sigma d W_{t}+\alpha_{t} d t$, starting at $X_{0}=x_{0}$. Here $W_{t}$ is a Brownian motion, $L$ is a cost for changing states, and $V$ relates to the state cost. All players solve the same stochastic control problem but differ in their initial characteristic $x_{0}$ distributed under a given probability measure $m_{0}$.

The criterion depends on the population density $m$ and this dependence models the interactions between players but reflects the insignificance of the individual influence (the agent is atomized in the economy). This aspect will also be used to traduce a scale effect and externalities. Each player chooses a rational strategy taking into account its own parameters but more especially the global distribution of agents $m$.

Mathematically, a mean field equilibrium (i.e. Nash point for an infinite number of players), leads to a PDE system coupling an Hamilton-Jacobi-Bellman equation and a Kolmogorov 
equation (see Ref. [12]):

$$
\left\{\begin{array}{l}
\partial_{t} m-\frac{\sigma^{2}}{2} \Delta m+\operatorname{div}\left(-\partial_{p} H(x, \nabla v) m\right)=0 \\
\partial_{t} v+\frac{\sigma^{2}}{2} \Delta v-H(x, \nabla v)=-V[m],
\end{array}\right.
$$

where $H$ is the Legendre transform of $L$ (see more details in Section 2.5). The MFG are suitable to describe large systems e.g., population dynamics (Ref. [9]), group interactions, etc. ..., in a stylized mathematical way amenable to numerical studies.

In this paper we focus on an application of MFG. The model enables us to design and test an efficient algorithm that finds the corresponding optimal points. The origins of the algorithm are in the field of quantum chemistry (see Ref. [14, 15, 21]).

We will proceed as follows: in Section 2 we present a model for the choice of insulation technology of households. We define the optimization problem and prove an existence result using a penalized problem. Then we introduce Eq. (1) using the optimal control framework. Section 3 is devoted to numerical setting and to the description of the algorithm. Finally, in Section 4, we show the efficiency of our method on some simulations. In particular we obtain a result showing the bundling due to the positive externality, and explore numerically the multiplicity of equilibria.

\section{The Model}

As mentioned before, our aim is not to give an exhaustive economic model, but to document qualitative behaviors related to the MFG model and propose an adapted numerical method. We choose to work in a continuous time setting, i.e. on the time interval $[0, T]$ where the MFG gives a very convenient characterization of a dynamic equilibrium. We look at a large economy and consider a continuum of consumer agents. Each agent is a household arbitrating between heating or better insulating their home. We next describe the modeling of the agents and their action.

\subsection{The agents}

Two important characteristics are assumed. First, any agent (household) owns exactly one house and cannot move to another before the horizon $T$. Secondly, we consider that the households are anonymous (i.e they have similar accommodations and preferences) but possibly different insulation levels. One of our goals is to find a dynamic equilibrium when there is the possibility of a technological transition (from energy consumption to insulation). Introducing externalities and economy of scale impacts the strategy of the households. Intuitively, we deal with a model where two particular behaviors are expected: either all agents continue heating their home or they all decide to insulate it.

Thus, each agent is associated to an insulation level, denoted by $x \in[0,1]$, which fully characterizes her state. The null insulation level $x=0$ corresponds to a house with only thin walls, whereas in the maximal insulation level $x=1$ all existent technologies of insulation are used. The dynamics of the agent corresponds to the following controlled process:

$$
d X_{t}=\sigma d W_{t}+\alpha_{t} d t+d N_{t}\left(X_{t}\right) .
$$

In this expression, the control variable is $\alpha_{t}$, which can be interpreted as the effort required to insulate. The agent optimizes its cost (described in the next section) with respect to $\alpha_{t}$. 
The term $W_{t}$ is a standard real valued Brownian motion centered in zero. The multiplicative factor corresponding to $W_{t}$ is the noise $\sigma$, which is related to the technological innovations and the climate (change of temperatures, storm, etc.); $\sigma$ is assumed to be a datum of the model. The variable $X_{t}$ is a diffusion process with reflexion. In the above formula, the reflexion part has been denoted by $N_{t}\left(X_{t}\right)$. In our model, this guarantees that the process stays in $[0,1]$. We refer to Ref. [5] for additional details about this formulation. The initial density of agents, denoted by $m_{0}$, is given; it describes agents' ownership of insulation material at $t=0$, and corresponds to the density of the initial variable $X_{0}$.

Remark 1. In what follows, we consider the evolution of the density of agents $m(t,$.$) for$ $t \in[0, T]$. We recall that for the diffusion process $d Y_{t}=\sigma d W_{t}+\alpha_{t} d t$ in $\mathbb{R}$, with initial distribution $m_{0}$, the evolution of the law of $Y_{t}$ follows the forward Kolmogorov equation (see Ref. [7] for a proof):

$$
\partial_{t} m-\frac{\sigma^{2}}{2} \Delta m+\operatorname{div}(\alpha m)=0 \text { in } \mathbb{R} \times(0, T], m(0, .)=m_{0}(.) .
$$

or, equivalently, for every test-function $\psi$ :

$$
\int_{\mathbb{R}} \psi(y) m(t, y) \mathrm{d} y=\int_{\mathbb{R}} \mathbb{E}\left(\psi\left(Y_{t}^{y}\right)\right) m_{0}(y) \mathrm{d} y .
$$

The reflexive part of process $X_{t}$ leads to the same equation in $[0,1] \times(0, T]$ with homogeneous Neumann boundary conditions $m^{\prime}(t, 0)=m^{\prime}(t, 1)=0$ for all $t \in(0, T)$. In other contexts this equation is also called the controlled Fokker-Planck equation.

We next model, through a cost functional to be minimized, how the agents control their insulation level.

\subsection{The costs}

In our model, any agent is assumed to minimize a cost functional composed of three terms: the cost of acquiring insulation, the insulation maintenance cost and the heating cost.

The cost of acquiring insulation is modeled as the cost to change the state that we take quadratic in the control variable:

$$
h\left(\alpha_{t}\right):=\frac{\alpha_{t}^{2}}{2} .
$$

The second term is a state cost, namely the insulation maintenance cost:

$$
g(t, x, m):=\frac{c_{0} x}{c_{1}+c_{2} m(t, x)},
$$

with $c_{0}, c_{1}$ and $c_{2}$ are some positive constants. In this setting, this term is increasing with respect to $x$ which means that the higher is the insulation level the higher is the maintenance cost. On the other hand, it is decreasing with respect to the global density of agents $m$. This property is related to two economical concepts: economy of scale and positive externality. Basically, this term encourages the agents to do similar choices: higher the number of players having chosen the same insulation level, lower its cost.

Finally, the heating cost is taken into account by introducing the third term:

$$
f(t, x):=p(t)(1-\beta x)
$$


In this definition, $\beta \in[0,1]$ is given, and $p(t)$ stands for the unit price of energy (electricity, gas, ...) that may depend on time; by unit we mean the price of energy for a quantity needed to heat a not insulated house. Various forms of this price are studied in the last section (simulations). The factor $(1-\beta x)$ is introduced to take into account the discount rate of the heating price with respect to the insulation level. Let us introduce the notation:

$$
\phi(m)(t):=\int_{0}^{1}(f(t, x)+g(t, x, m)) m(t, x) d x .
$$

The addition of these three costs will constitute the functional to be minimized, as we will see in the next paragraph.

\subsection{Minimization problem and MFG}

We are now in the position to write the minimization problem we consider in this paper. A first formulation is:

$$
\left\{\begin{array}{l}
\inf _{\alpha} \mathbb{E}\left[\int_{0}^{T} h\left(\alpha\left(t, X_{t}^{x}\right)\right)+V[m]\left(X_{t}^{x}\right) d t\right] \\
d X_{t}^{x}=\alpha_{t} d t+\sigma d W_{t}+d N_{t}\left(X_{t}^{x}\right), \\
X_{0}^{x}=x
\end{array}\right.
$$

$V=\phi^{\prime}$ (see Ref. [11]). For the sake of simplicity, we consider that there is no other constraint on the control variable. This system corresponds to a Lagrangian formulation, dealing with the microscopic scale (the agent). As mentioned in Remark (1), it is standard to write the Eulerian formulation of the problem:

$$
\left\{\begin{array}{l}
\inf _{\alpha} \int_{0}^{T}\left[\int_{0}^{1} \frac{\alpha(t, x)^{2}}{2} m(t, x) d x+\phi(m)(t)\right] d t \\
\partial_{t} m-\frac{\sigma^{2}}{2} \Delta m+\operatorname{div}(\alpha m)=0, m(0, .)=m_{0}(.),
\end{array}\right.
$$

with boundary conditions: $m^{\prime}(., 0)=m^{\prime}(., 1)=0, \alpha(., 0)=\alpha(., 1)=0$. Our work is related to the optimal control of the forward Kolmogorov equation. In this model, the agents are rational and they have rational expectations: in the minimization problem, the households see $m(t,$.$) as given for every t$ in $[0, T]$. A solution of this problem will present many similarities with an equilibrium with rational expectations.

Writing our problem as a MFG problem with a finite horizon (as in Ref. [12]) means that the critical points of problem (5) correspond to mean field equilibria. As recalled in the introduction, these are approximations of Nash equilibria of a $N$-players game, for large values of $N$. This tool enables to handle a continuum of agents, hence atomized in the economy. Consequently, there is no need to build a representative agent. We consider all the agents, from the macroscopic point of view. Before writing the critical points equations, we investigate the existence of a minimizer for a penalized problem.

\subsection{Existence}

Let $\Omega$ be the space domain; we will consider as important cases $(0,1)$ with Neumann boundary conditions and the $d$-dimensional thorus $\mathbb{T}^{d}$. 
Let us define the time-space domain $Q:=[0, T] \times \Omega$, a nonnegative continuous function $\eta: Q \times \mathbb{R} \rightarrow \mathbb{R}_{+}$, and the function:

$$
\begin{aligned}
\Phi: \mathcal{M}_{b}^{a c}\left(\mathbb{R}_{+}\right) \times[0, T] & \rightarrow \mathbb{R} \\
(m, t) & \mapsto \Phi(m)(t):=\int_{\Omega} \eta(t, x, m(x)) d x,
\end{aligned}
$$

where $\mathcal{M}_{b}^{a c}\left(\mathbb{R}_{+}\right)$denotes the space of Borel measures supported on $\Omega$, which are absolutely continuous with respect to the Lebesgue measure. Note that $\phi$ introduced in Section 2.2 is a particular case of $\Phi$ above.

We refer the reader to Ref. [22] (Chap XVIII, $\S 4.4$ ) for the precise functional space setting. When $\Omega=(0,1)$ with homogenous Neumann boundary conditions or when $\Omega=\mathbb{T}^{d}$ we take $V=H^{1}(\Omega)$. For mixed Dirichlet-Neumann or more general boundary conditions the natural space $V$ satisfies $H_{0}^{1}(\Omega) \subset V \subset H^{1}(\Omega)$. In all cases existence of a solution of the evolution problem is ensured for $\alpha \in L^{\infty}\left(0, T ; W^{1, \infty}(\Omega)\right)$. Denote $W(0, T):=\left\{u: u \in L^{2}(0, T ; V), \frac{d u}{d t} \in\right.$ $\left.L^{2}\left(0, T ; V^{\prime}\right)\right\}$. One can prove that $W(0, T)$ is continuously included in $C^{0}\left(0, T ; L^{2}(\Omega)\right)$. We refer to Ref. [23] (Chap 2. $\S 5.5$ ) for additional discussions on alternative functional settings.

We are interested in the following minimization problem:

$$
\left\{\begin{array}{l}
\inf _{\alpha} \int_{0}^{T}\left[\int_{\Omega}|\alpha(t, x)|^{2} m(t, x) d x+\Phi\left(m_{t}\right)(t)\right] d t \\
\partial_{t} m-\frac{\sigma^{2}}{2} \Delta m=-\operatorname{div}(\alpha m), m(0, .)=m_{0}(.),+ \text { boundary conditions. }
\end{array}\right.
$$

For notational convenience we consider in what follows the case $\frac{\sigma^{2}}{2}=1$. We introduce, as in Ref. [1] and Ref. [2], the transformation $q=\alpha m$ i.e. the functions

$$
\psi(a, b):=\left\{\begin{array}{l}
\frac{|a|^{2}}{b} \text { if }(a, b) \in \mathbb{R}^{d} \times \mathbb{R}_{+}^{*} \\
+\infty \text { otherwise }
\end{array}\right.
$$

and

$$
K(q, m):=\int_{Q} \psi(q, m)+\int_{0}^{T} \Phi\left(m_{t}\right)(t) d t .
$$

Following Ref. [2], we can write $K$ in a simpler form:

$$
K(q, m)=\left\{\begin{array}{c}
\int_{0}^{T}\left[\left(\int_{\Omega} \frac{|q|^{2}}{m}\right)+\Phi\left(m_{t}\right)(t)\right] d t \\
\quad:=\int_{0}^{T}\left[\int_{\Omega}|\alpha(t, x)|^{2} m(t, x) d x+\Phi\left(m_{t}\right)(t)\right] d t \text { if } q=\alpha m \\
+\infty \text { otherwise, }
\end{array}\right.
$$

Then (6) can be rewritten:

$$
\left\{\begin{array}{l}
\inf _{(q, m) \in B} K(q, m) \\
B:=\left\{(q, m): q \in L^{2}(Q), m \text { weak solution of } \partial_{t} m-\Delta m=-\operatorname{div}(q)\right. \\
\left.m(0, .)=m_{0}(.) \in L^{2}(\Omega), m \in W(0, T)\right\}
\end{array}\right.
$$

Since we have no information about the convexity of $\Phi$, we introduce the penalized problem:

$$
\inf _{(q, m) \in B} K_{\varepsilon}(q, m):=K(q, m)+\varepsilon\|q\|_{2}^{2},
$$

where $\|\cdot\|_{2}$ denotes the $L^{2}(Q)$-norm. 
Theorem 2.1. If $m_{0} \in L^{2}(\Omega)$, then the minimization problem (8) admits a solution $\left(q_{\varepsilon}, m_{\varepsilon}\right) \in$ $B$ with $q_{\varepsilon} \in L^{2}(Q)$, moreover:

$\lim _{\varepsilon \rightarrow 0}\left(\min _{(q, m) \in B} K_{\varepsilon}(q, m)\right)=\inf _{(q, m) \in B} K(q, m)$.

Proof. The proof proceeds in several steps that we describe below. Let $\left(q_{n}, m_{n}\right)$ be a minimizing sequence of problem (8).

Step 1: bounds and convergence of $q_{n}$. Obviously $\left(q_{n}, m_{n}\right)$ is in the domain of $K$. There exists a positive constant $\tilde{C}$ such that $\tilde{C} \geqslant K_{\varepsilon}\left(q_{n}, m_{n}\right) \geqslant \varepsilon\left\|q_{n}\right\|_{2}^{2}$. The sequence $q_{n}$ is bounded in $L^{2}(Q)$, consequently there exists a subsequence (denoted again by $q_{n}$ ) which converges weakly to a limit $q \in L^{2}(Q)$. Note also that for all $n, \operatorname{div}\left(q_{n}\right)$ is bounded in $L^{2}\left(0, T ; V^{\prime}\right)$.

Step 2: convergence of $m_{n}$. As shown in Ref. [13], since $\operatorname{div}\left(q_{n}\right) \in L^{2}\left(0, T ; V^{\prime}\right)$ and $m_{0} \in L^{2}(\Omega)$, then $m_{n} \in W(0, T)$. Moreover $\operatorname{div}\left(q_{n}\right)$ is bounded in $L^{2}\left(0, T ; V^{\prime}\right)$ thus $m_{n}$ is bounded in $W(0, T)$. We want to prove that up to the extraction of a subsequence, $m_{n}$ converges strongly to a limit $m$ in $L^{2}(Q)$. The key properties are the injections:

$$
V \subset L^{2}(\Omega) \subset V^{\prime},
$$

where both injections are continuous and the first one is compact. Since the injection $V \subset$ $L^{2}(\Omega)$ is compact one finds that $W(0, T) \subset L^{2}(Q)$ is also compact, cf. Ref. [20] for a rigorous proof. Then there exists $m \in W(0, T)$ and subsequence $m_{n}$ such that $m_{n}$ converges to $m$ weakly in $W(0, T)$ and strongly in $L^{2}(Q)$. Finally, there is another subsequence (again denoted by $m_{n}$ ) converging a.e to $m$.

Note that since $q_{n} \rightarrow q$ in $L^{2}(Q)$ and $m_{n} \rightarrow m$ in $W(0, T)$ then $m$ verifies the weak form of the equation $\partial_{t} m-\Delta m=-\operatorname{div}(q)$ thus $(q, m) \in B$.

Step 3: taking limits. The last remaining point is the limit of the functional. Thanks to the lower semicontinuity of the $L^{2}(Q)$-norm and the left hand side of $K$ (see Ref. [2]), the convergence a.e. of $m_{n}$ to $m$, the continuity of $\eta$ and Fatou's Lemma, we have:

$$
\begin{aligned}
K_{\varepsilon}(q, m) & =\lim _{n \rightarrow \infty} K_{\varepsilon}\left(q_{n}, m_{n}\right) \\
& =\liminf _{n \rightarrow \infty} K_{\varepsilon}\left(q_{n}, m_{n}\right) \\
& \geqslant \int_{Q} \frac{|q|^{2}}{m}+\int_{0}^{T} \Phi(m)+\varepsilon\|q\|_{2}^{2}=K_{\varepsilon}(q, m),
\end{aligned}
$$

which shows that $(q, m)$ is a minimizer of problem $(7)$.

Finally, let us prove the last assertion of the theorem.

Step 4: limit of the penalized problems. Let $\left(q_{\varepsilon}, m_{\varepsilon}\right)$ be a minimizer of problem (8), then:

$$
\inf _{(q, m) \in B} K(q, m) \leqslant K\left(q_{\varepsilon}, m_{\varepsilon}\right)+\varepsilon\left\|q_{\varepsilon}\right\|_{2}^{2} \leqslant K(q, m)+\varepsilon\|q\|_{2}^{2}, \forall(q, m) \in B
$$

Let us take the limit when $\varepsilon$ converges to 0 :

$$
\inf _{(q, m) \in B} K(q, m) \leqslant \liminf _{\varepsilon \rightarrow 0} K_{\varepsilon}\left(q_{\varepsilon}, m_{\varepsilon}\right) \leqslant \limsup _{\varepsilon \rightarrow 0} K_{\varepsilon}\left(q_{\varepsilon}, m_{\varepsilon}\right) \leqslant K(q, m), \forall(q, m) \in B
$$


Take the infimum in the right hand part of these inequalities and obtain:

$$
\inf _{(q, m) \in B} K(q, m) \leqslant \liminf _{\varepsilon \rightarrow 0} K_{\varepsilon}\left(q_{\varepsilon}, m_{\varepsilon}\right) \leqslant \limsup _{\varepsilon \rightarrow 0} K_{\varepsilon}\left(q_{\varepsilon}, m_{\varepsilon}\right) \leqslant \inf _{(q, m) \in B} K(q, m),
$$

hence the conclusion.

Remark 2. Note that for all $\varepsilon>0$, if $\left(q_{\varepsilon}, m_{\varepsilon}\right)$ is a minimizer of problem (8), the following assertions are equivalent:

1. problem (7) admits a solution $(q, m) \in B$ with $q \in L^{2}(Q)$,

2. $\exists M>0$ such that $\forall \varepsilon>0,\left\|q_{\varepsilon}\right\|_{2}<M$.

To see that $1 \Rightarrow 2$ let $q$ be optimal in (7); from the optimality of $q$ and $q_{\varepsilon}$ one obtains that for all $\varepsilon>0,\left\|q_{\varepsilon}\right\|_{2} \leqslant\|q\|_{2}$. Let us now prove $2 \Rightarrow 1$. Since $\left(q_{\varepsilon}\right)_{\varepsilon>0}$ is uniformly bounded in $L^{2}(Q)$, we have:

$$
\lim _{\varepsilon \rightarrow 0} \varepsilon\left\|q_{\varepsilon}\right\|_{2}=0 .
$$

Then, using Theorem (2.1) we conclude that $\left(q_{\varepsilon}, m_{\varepsilon}\right)$ is a minimizing sequence for $K$. Proceeding analogously to the proof of the previous theorem, we obtain that there exists a subsequence $\left(q_{\varepsilon_{n}}, m_{\varepsilon_{n}}\right)$ such that $q_{\varepsilon_{n}}$ converges weakly to a limit $w \in L^{2}(Q)$ and $m_{\varepsilon_{n}}$ converges strongly to $m_{w} \in L^{2}(Q)$. Furthermore, $\left(w, m_{w}\right)$ is a solution of problem (7) and:

$$
\left\|q_{\varepsilon_{n}}\right\|_{2} \leqslant\|w\|_{2}
$$

The weak convergence of $q_{\varepsilon_{n}}$ and the previous inequality successively yield to:

$$
\lim _{n \rightarrow \infty} \int_{Q}\left(q_{\varepsilon_{n}}-w\right)^{2}=\lim _{n \rightarrow \infty} \int_{Q} q_{\varepsilon_{n}}^{2}-w^{2} \leqslant 0,
$$

so that $q_{\varepsilon_{n}}$ converges strongly in $L^{2}(Q)$.

\subsection{Optimality conditions}

In this section, we describe in a formal way how to obtain optimality conditions for problem (5). A rigorous mathematical derivation is sketched in Ref. [10, 11, 12]. Let us start by introducing the notation:

$$
J(m, \alpha):=\int_{0}^{T}\left[\int_{0}^{1} \frac{\alpha(t, x)^{2}}{2} m(t, x) d x+\phi(m)(t)\right] d t .
$$

Let us now note that (3) can be written in the weak form as:

$$
\int_{0}^{1}\left(v(T, .) m(T, .)-v(0, .) m_{0}(x)\right)=\int_{0}^{T}\left[\phi(m)+\int_{0}^{1}\left(\partial_{t} v+\frac{\sigma^{2}}{2} \Delta v+\alpha \cdot \nabla v\right) m\right]
$$

for every $v \in C_{c}^{\infty}([0,1] \times[0, T])$. We define the Lagrangian of problem (5):

$$
\begin{array}{r}
\mathcal{L}(m, \alpha, v):=J(m, \alpha)+\int_{0}^{T}\left[\phi(m)+\int_{0}^{1}\left(\partial_{t} v+\frac{\sigma^{2}}{2} \Delta v+\alpha \cdot \nabla v\right) m\right] \\
-\int_{0}^{1} v(T, .) m(T, .)+\int_{0}^{1} v(0, .) m_{0}
\end{array}
$$


The minimization problem (5) can be rewritten as a saddle-point problem:

$$
\inf _{(m, \alpha)} \sup _{v} \mathcal{L}(m, \alpha, v)
$$

and the conditions characterizing the saddle-point of $\mathcal{L}$ read as (3) together with:

$$
\begin{array}{r}
\alpha=-\nabla v, \mathrm{~m}-\text { a.e. } \\
\partial_{t} v+\frac{1}{2} \alpha^{2}+\alpha \cdot \nabla v+\frac{\sigma^{2}}{2} \Delta v=-\phi^{\prime}(m), \\
v(T, .)=0 .
\end{array}
$$

We obtain the critical point equations that include, in addition to the equation for $m$, the formula for the optimal control $\alpha:=-\nabla v$ and the backward Hamilton-Jacobi-Bellman equation for $v$

$$
\begin{array}{r}
\partial_{t} v+\frac{\sigma^{2}}{2} \Delta v-\frac{1}{2}|\nabla v|^{2}=-\phi^{\prime}(m) \\
v(T, .)=0 .
\end{array}
$$

Remark 3. By introducing the Hamiltonian of the system, defined as the Legendre transform of the changing state cost: $H(x, p)=\sup _{\alpha} \alpha p-\frac{\alpha^{2}}{2}=\frac{p^{2}}{2}$, it is easy to see that the optimality system of critical points equations reads:

$$
\begin{array}{r}
\partial_{t} m-\frac{\sigma^{2}}{2} \Delta m+\operatorname{div}(\alpha m)=0, m(0, .)=m_{0}, \\
\alpha=-\partial_{p} H(x, \nabla v), \\
\partial_{t} v+\frac{\sigma^{2}}{2} \Delta v-H(x, \nabla v)=-\phi^{\prime}(m), v(T, .)=0 .
\end{array}
$$

Finally, we introduce a mean field equilibrium as the solution of the mean field system:

$$
\left\{\begin{array}{l}
\partial_{t} m-\frac{\sigma^{2}}{2} \Delta m+\operatorname{div}(\alpha m)=0, m(0, .)=m_{0}, m^{\prime}(.0)=m^{\prime}(., 1)=0 \\
-\nabla v=\alpha \\
\partial_{t} v+\frac{\sigma^{2}}{2} \Delta v+\frac{\alpha^{2}}{2}+\alpha \cdot \nabla v=-\phi^{\prime}(m), v(T, .)=0
\end{array}\right.
$$

consisting in two coupled Partial Differential Equations (PDEs), one being backward (the Hamilton-Jacobi-Bellman equation) and the other one being forward (the Kolmogorov equation). This coupling will be important for the choice of the numerical method.

\subsection{Some remarks}

We conclude this section with some observations about the modeling with the "tool" MFG. The MFG seem to be particularly adapted to describe a situation that combines two economical ideas, positive externality and scale effect. In our model, we introduced a positive externality (if one insulates better her home, her neighbor has a better insulation of her apartment). We saw that there is a clear incentive on any agent to choose some insulation level.

This can be understood considering the form of the insulation maintenance cost, which is decreasing in the global distribution of agents $m$. In addition, the negative slope in $m$ means that there is also an economy of scale in terms of savings that a firm obtains due 
to expansion (think of the maintenance costs). We will see in the last section (using some numerical simulations) that people indeed stay together i.e., tend to agglomerate.

Let us add some comments about the characteristics of the model. First of all, recall that the model is in continuous time and space. This is adapted to the study of transition effects (in particular the possibility for the population to change the consumed technology). Moreover the modeling of an infinite number of agents is strongly linked with the atomization of the agents in the economy. The MFG enables to handle such a situation. On the one hand every household is atomized (she takes into account that her action has no influence on the global density) and on the other hand she has rational expectations (thus she sees the global density as a datum), and the Nash point approximation leads consequently to a non-cooperative equilibria.

To conclude our remarks, we emphasize that our model is a stylized model from the industrial point of view. It is not completely realistic (heating price, maintenance cost) nor a simplification of statistical data. A work of calibration can be done, but is beyond the scope of the present paper. Before some comments on the behavior of a population of agents with this modeling, let us give the algorithm that will be used for simulations.

\section{Numerical Simulations}

In this section, we describe the algorithm that we use to solve our problem. This algorithm is an adaptation of a procedure initially developed in the field of quantum chemistry Ref. [19, 21] (see also Ref. [14, 15]) following an approach introduced by Krotov Ref. [8]. It has recently been extended to some transport problems in Ref. [4] (see also Ref. [9]). For a general presentation of this algorithm, see Ref. [18]. This approach shows excellent results on non-convex problems, where usual gradient methods fail to converge.

Before presenting the optimization algorithm, we introduce a relevant discretization of the problem. Even if what follows easily generalizes to $2 \mathrm{D}$ and $3 \mathrm{D}$ situations, we focus in the rest of the paper on the 1D-case for its simplicity and the consistence with the initial dimension of the problem. Given a matrix $C$, we denote by $C^{*}$ its transpose.

Let us consider two positive integers $M, N$. We consider here the case of the bounded space domain $[0,1]$ for (3). We define the time and space steps by $d t=\frac{1}{N}$ and $d x=\frac{1}{M}$ and denote for $j=0, \ldots, M, i=0, \ldots, N$ by $m_{j}^{i}$ the numerical approximations of $m(i . d t, j . d x)$. For reasons that will appear later, the discrete control is defined at the points $(i . d t,(j+1 / 2) . d x)$, and is therefore denoted by $\alpha_{j+1 / 2}^{i}$. We shall also use the notations $\alpha^{i}=\left(\alpha_{j+1 / 2}^{i}\right)_{j=1 \ldots M-1}$ and $m^{i}=\left(m_{j}^{i}\right)_{j=1 \ldots M-1}$. Finally, we denote by $\phi_{j}\left(m^{i}\right)$ the approximation of the maintenance cost $\phi(m, t, x)$ at the point $(m(i . d t, j . d x), i . d t, j . d x)$. Remember that the total mass of $[0,1]$ is constant in time, so that at the discrete level, we impose the homogeneous Neumann boundary condition expressed by $\alpha_{1 / 2}^{i}=\alpha_{M-1 / 2}^{i}=0$, for $i=0 \ldots N-1$.

\subsection{Discretized cost functional}

From now on, $\alpha$ stands for the discrete control $\left(\alpha_{j+1 / 2}^{i}\right)_{i, j}$ and $g\left(m^{i}\right)$ is the real number defined by $g\left(m^{i}\right)=d x \sum_{j=1}^{M-1} \Phi_{j}\left(m^{i}\right)$ where $\Phi_{j}\left(m^{i}\right)=\phi_{j}\left(m^{i}\right) m_{j}^{i}$.

We consider the following discrete version of the cost functional $J$ : 


$$
\begin{aligned}
J_{d t, d x}(\alpha): & =d t . d x \sum_{i=0}^{N-1} \sum_{j=1}^{M-1}\left(\frac{1}{2} r_{j}\left(\alpha^{i}\right) m_{j}^{i}+\Phi_{j}\left(m^{i}\right)\right) \\
& =d t \sum_{i=0}^{N-1}\left(\frac{1}{2}\left\langle m^{i}, r\left(\alpha^{i}\right)\right\rangle+g\left(m^{i}\right)\right)
\end{aligned}
$$

where $\langle\cdot, \cdot\rangle$ is the scalar product on $\mathbb{R}^{M-1}$ defined by:

$$
\langle u, v\rangle=d x \sum_{j=1}^{M-1} u_{j} v_{j} .
$$

The vector $r\left(\alpha^{i}\right)=\left(r_{j}\left(\alpha^{i}\right)\right)_{j=1 \ldots M-1}$ is defined from $\alpha^{i}$ by:

$$
r_{j}\left(\alpha^{i}\right)=\frac{\left(\alpha_{j-1 / 2}^{i}\right)^{2}+\left(\alpha_{j+1 / 2}^{i}\right)^{2}}{2},
$$

and corresponds to use a trapezoid rule to approximate $|\alpha|^{2}$ in the integral of (10).

\subsection{Numerical scheme for the controlled Kolmogorov equation}

The preservation of the positivity of $m$ at the discrete level appears in numerical simulation as a crucial issue, especially for small values of $\sigma$. Indeed, the concavity of $J$ with respect to $m$ leads to numerical instabilities when using schemes which do not possess this property, independently of their order of accuracy. This fact motivates the use of a low order Godunov scheme for the advective part of (3) which ensures both small computational cost and positivity of the numerical solutions.

Remark 4. In addition, this framework enables us to avoid the introduction of Lagrange multipliers corresponding to the constraint $m \geq 0$.

We are now in the position to define the numerical solver of (3). Starting from $m_{j}^{0}=$ $m_{0}(j . d x), m_{j}^{i}$ is computed iteratively for $j=1 \ldots M-1$ by:

$$
\begin{aligned}
m_{j}^{i+1}= & m_{j}^{i}+\frac{\sigma^{2}}{2} \frac{d t}{d x^{2}}\left(m_{j+1}^{i}-2 m_{j}^{i}+m_{j-1}^{i}\right) \\
& -\frac{d t}{d x}\left(m_{j+1 / 2}^{i} \alpha_{j+1 / 2}^{i}-m_{j-1 / 2}^{i} \alpha_{j-1 / 2}^{i}\right) .
\end{aligned}
$$

In this equation, the terms $m_{j+1 / 2}^{i}$ and $m_{j-1 / 2}^{i}$ of the advective part are defined according to a Godunov scheme, i.e. using up-winding:

$$
m_{j+1 / 2}^{i}=\left\{\begin{array}{cll}
m_{j+1}^{i} & \text { if } & \alpha_{j+1 / 2}^{i}<0 \\
m_{j}^{i} & \text { if } & \alpha_{j+1 / 2}^{i} \geq 0
\end{array}\right.
$$

For more details about this type of schemes, we refer to Ref. $[16,6]$.

To simplify our notations, we rewrite (16) as:

$$
m^{i+1}=\left(A+B\left(\alpha^{i}\right)\right) m^{i},
$$


Here $A$ corresponds to the identity matrix plus the discrete Laplace operator, i.e. the first two terms of the right hand-side of (16) and $B$ is associated to the advective part, i.e. the last term of the right hand-side of (16). The choice of such a scheme ensures the positivity of the density at the discrete level, under the condition of the following Lemma:

Lemma 3.1. For $i=1, \ldots, N$, if for every $j=1, \ldots, M-1, m^{i} \geq 0$ and:

$$
\left|\alpha_{j+1 / 2}^{i}\right| \leq \lambda:=\frac{d x}{2 d t}-\frac{\sigma^{2}}{2} \frac{1}{d x},
$$

then $m^{i+1} \geq 0$.

Proof. Let us fix $i, 1 \leq i \leq N$, and $j, 1 \leq j \leq M-1$. Suppose that (18) holds, $\alpha_{j+1 / 2}^{i} \geq 0$ and $\alpha_{j-1 / 2}^{i} \geq 0$. Thanks to $(16), m_{j}^{i+1}$ reads as a linear combination of $m_{j+1}^{i}, m_{j}^{i}$ and $m_{j-1}^{i}$, with coefficients $\frac{\sigma^{2}}{2} \frac{d t}{d x^{2}}, 1-\sigma^{2} \frac{d t}{d x^{2}}-\alpha_{j+1 / 2}^{i} \frac{d t}{d x}$ and $\frac{\sigma^{2}}{2} \frac{d t}{d x^{2}}+\alpha_{j-1 / 2}^{i} \frac{d t}{d x}$. Condition (18) then guarantees that these coefficients are positive, so that the linear combination is indeed a convex combination. Other cases shall be dealt similarly.

\subsection{Adjoint state}

In the approach we follow, a crucial role is played by the adjoint state $v^{i}=\left(v_{j}^{i}\right)_{j=1 \ldots M-1}$ that is defined iteratively for $i=0 \ldots N$, by the backward propagation (Hamilton-Jacobi-Bellman $\mathrm{PDE})$ :

$$
\begin{aligned}
v^{N} & =0, \\
v^{i} & =\left(A^{*}+B^{*}\left(\alpha^{i}\right)\right) v^{i+1}+\frac{d t}{2} r\left(\alpha^{i}\right)+d t \Phi^{\prime}\left(m^{i}\right) .
\end{aligned}
$$

where $\Phi^{\prime}\left(m^{i}\right)$ is the vector with components $\Phi_{j}^{\prime}\left(m^{i}\right)$. This variable is the discrete version of the Lagrange multiplier defined by (11-13).

\subsection{Variations in $J_{d t, d x}$}

We present now the algebraic manipulations at the heart of the monotonic schemes. Let us consider two controls $\alpha$ and $\alpha^{\prime}$ and the corresponding solutions $\left(m^{i}\right)_{i=0 \ldots N}$ and $\left(m_{i}^{\prime}\right)_{i=0 \ldots N}$ of (16). In what follows, the adjoint $v=\left(v_{i}\right)_{i=0 \ldots N}$ corresponds to $\alpha$. One has:

$$
\begin{array}{r}
J_{d t, d x}\left(\alpha^{\prime}\right)-J_{d t, d x}(\alpha)=\frac{d t}{2} \sum_{i=0}^{N-1}\left\langle m^{i}, r\left(\alpha^{i}\right)-r\left(\alpha^{i}\right)\right\rangle+\frac{d t}{2} \sum_{i=0}^{N-1}\left\langle m^{\prime i}-m^{i}, r\left(\alpha^{i}\right)\right\rangle \\
+\sum_{i=0}^{N-1}\left\langle m^{\prime i+1}-m^{i+1}, v^{i+1}\right\rangle-\left\langle m^{\prime i}-m^{i}, v^{i}\right\rangle \\
+d t \sum_{i=0}^{N-1} g\left(m^{i}\right)-g\left(m^{i}\right) .
\end{array}
$$

A crucial fact is that, for $j=1, \ldots, M-1$, functions $\Phi_{j}$ are concave in $m$. Consequently, we get:

$$
\sum_{i=0}^{N-1} g\left(m^{i}\right)-g\left(m^{i}\right) \leq \sum_{i=0}^{N-1}\left\langle\Phi^{\prime}\left(m^{i}\right), m^{i}-m^{i}\right\rangle,
$$


where $\Phi^{\prime}$ is defined in Section 3.3. Combining (20) with (21) we can write:

$$
\begin{array}{r}
J_{d t, d x}\left(\alpha^{\prime}\right)-J_{d t, d x}(\alpha) \leq \frac{d t}{2} \sum_{i=0}^{N-1}\left\langle m^{i}, r\left(\alpha^{i}\right)-r\left(\alpha^{i}\right)\right\rangle \\
+\sum_{i=0}^{N-1}\left\langle\left(A+B\left(\alpha^{i}\right)\right) m^{i}-\left(A+B\left(\alpha^{i}\right)\right) m^{i}, v^{i+1}\right\rangle \\
+\sum_{i=0}^{N-1}\left\langle m^{i}-m^{i},-v^{i}+\frac{d t}{2} r\left(\alpha^{i}\right)+d t \Phi^{\prime}\left(m^{i}\right)\right\rangle .
\end{array}
$$

We finally obtain:

$$
\begin{array}{r}
J_{d t, d x}\left(\alpha^{\prime}\right)-J_{d t, d x}(\alpha) \leq \frac{d t}{2} \sum_{i=0}^{N-1}\left\langle m^{i}, r\left(\alpha^{i}\right)-r\left(\alpha^{i}\right)\right\rangle \\
+\sum_{i=0}^{N-1}\left\langle\left(B\left(\alpha^{i}\right)-B\left(\alpha^{i}\right)\right) m^{i}, v^{i+1}\right\rangle .
\end{array}
$$

This inequality can also be expressed through the formula:

$$
J_{d t, d x}\left(\alpha^{\prime}\right)-J_{d t, d x}(\alpha) \leq d t . d x \sum_{i=0}^{N-1} \sum_{j=1}^{M-2} \Delta_{j}^{i}\left(\alpha^{\prime}, \alpha\right),
$$

where:

$$
\begin{gathered}
\Delta_{j}^{i}\left(\alpha^{\prime}, \alpha\right)=\frac{m_{j}^{i}+m_{j+1}^{i}}{2}\left(\frac{\left(\alpha_{j+1 / 2}^{i}\right)^{2}-\left(\alpha_{j+1 / 2}^{i}\right)^{2}}{2}\right) \\
+\left(m_{j+1 / 2}^{i} \alpha_{j+1 / 2}^{i i}-{\widetilde{m^{\prime}}}_{j+1 / 2}^{i} \alpha_{j+1 / 2}^{i}\right)\left(\frac{v_{j+1}^{i+1}-v_{j}^{i+1}}{d x}\right) .
\end{gathered}
$$

In this equation we introduced

$$
{\widetilde{m^{\prime}}}_{j+1 / 2}^{i}=\left\{\begin{array}{ccc}
m_{j+1}^{i} & \text { if } & \alpha_{j+1 / 2}^{i}<0 \\
m_{j}^{i i} & \text { if } & \alpha_{j+1 / 2}^{i} \geq 0
\end{array}\right.
$$

Note that inequality (23) disintegrates the variables of $J$ into a sum of local growth elements. This inequality that reads as a factorization enables to build a monotonic optimization method for functional $J$.

Remark 5. Given $\alpha$, note that the value of $m_{j+1 / 2}^{i}$ depends on the sign of $\alpha_{j}^{\prime i}$, so that $\alpha_{j}^{\prime i} \mapsto$ $\Delta_{j}^{i}\left(\alpha^{\prime}, \alpha\right)$ is a continuous, piecewise polynomial function.

Remark 6. Note that the strategy followed to obtain (23) applies for more general situations. Indeed, the two necessary requirements to make use of our method are the linearity of the dynamic and the concavity of the functional with respect to the state variable. We refer to Ref. [18] for more details. In particular other types of dependance of the cost with respect to $\alpha$ could be treated. 


\subsection{Optimization procedure}

\subsubsection{The method}

This section provides a brief summary of the optimization strategy we follow to solve our problem.

Given $\alpha$ and a positive real number $\theta$, we define $\alpha_{j+1 / 2}^{i}$ as a solution of

$$
\Delta_{j}^{i}\left(\alpha^{\prime}, \alpha\right)=-\theta \frac{m_{j}^{\prime i}+m_{j+1}^{\prime i}}{2}\left(\alpha_{j+1 / 2}^{\prime i}-\alpha_{j+1 / 2}^{i}\right)^{2} .
$$

According to Remark 5, this equation may have one, two or four roots, including the trivial one $\alpha_{j+1 / 2}^{\prime i}=\alpha_{j+1 / 2}^{i}$. When possible, we define $\alpha_{j+1 / 2}^{i}$ as the root of (25) that is closer to $\alpha_{j+1 / 2}^{i}$; otherwise we set $\alpha_{j+1 / 2}^{i}=\alpha_{j+1 / 2}^{i}$. Thus, the monotonicity of our algorithm is guaranteed.

Let us give the explicit formula corresponding to this procedure. We suppose that $\frac{m_{j}^{\prime i}+m_{j+1}^{\prime i}}{2}>$ 0 , otherwise the contribution of this term is zero for all choice of $\alpha_{j+1 / 2}^{i}$. We introduce

$$
\begin{array}{r}
\delta=\frac{2}{\theta+1}, \quad \nu_{j+1 / 2}^{i}=\frac{2 \hat{m}_{j+1 / 2}^{i}}{m_{j}^{\prime i}+m_{j+1}^{\prime i}}, \quad \widetilde{\nu}_{j+1 / 2}^{i}=\frac{2{\widetilde{m^{\prime}}}_{j+1 / 2}^{i}}{m_{j}^{\prime i}+m_{j+1}^{\prime i}}, \\
\hat{m}_{j+1 / 2}^{i i}=\left\{\begin{array}{ccc}
m_{j+1}^{\prime i} & \text { if } & \widetilde{m}_{j+1 / 2}^{\prime i}=m_{j}^{\prime i} \\
m_{j}^{\prime i} & \text { if } & \widetilde{m}_{j+1 / 2}^{\prime i}=m_{j+1}^{\prime i}
\end{array}\right.
\end{array}
$$

Consider now:

$$
\begin{aligned}
\xi_{j+1 / 2}^{i} & =(1-\delta) \alpha_{j+1 / 2}^{i}+\delta \widetilde{\nu}_{j+1 / 2}^{i} \frac{v_{j+1}^{i+1}-v_{j}^{i+1}}{d x}, \\
\beta_{j+1 / 2}^{i}= & \frac{-b_{j+1 / 2}^{i}-\operatorname{sign}\left(\alpha_{j+1 / 2}^{i}\right) \sqrt{\left(b_{j+1 / 2}^{i}\right)^{2}-4 a_{j+1 / 2}^{i} \cdot c_{j+1 / 2}^{i}}}{2 a_{j+1 / 2}^{i}},
\end{aligned}
$$

where sign is the function

$$
\operatorname{sign}(x)=\left\{\begin{array}{cll}
1 & \text { if } & x \geq 0 \\
-1 & \text { if } & x<0
\end{array}\right.
$$

and :

$$
\begin{aligned}
a_{j+1 / 2}^{i} & =1+\theta \\
b_{j+1 / 2}^{i} & =-2\left(\theta \alpha_{j+1 / 2}^{i}+\nu_{j+1 / 2}^{i} \frac{v_{j+1}^{i+1}-v_{j}^{i+1}}{d x}\right), \\
c_{j+1 / 2}^{i} & =(\theta-1)\left(\alpha_{j+1 / 2}^{i}\right)^{2}+2 \alpha_{j+1 / 2}^{i} \widetilde{\nu}_{j+1 / 2}^{i} \frac{v_{j+1}^{i+1}-v_{j}^{i+1}}{d x} .
\end{aligned}
$$

The control $\alpha_{j}^{i}$ can be expressed by :

$$
\alpha_{j+1 / 2}^{\prime i}=\left\{\begin{array}{lll}
\xi_{j+1 / 2}^{i} & \text { if } & \alpha_{j+1 / 2}^{i} \cdot \xi_{j+1 / 2}^{i} \geq 0 \\
\beta_{j+1 / 2}^{i} & \text { if } & \alpha_{j+1 / 2}^{i} \cdot \xi_{j+1 / 2}^{i}<0 .
\end{array}\right.
$$

Proposition 3.2. Under the condition (18), the scheme (27) ensures $J_{d t, d x}\left(\alpha^{\prime}\right) \leq J_{d t, d x}(\alpha)$ i.e., the functional $J_{d t, d x}$ decreases. 
Proof. The monotonicity is a trivial consequence of Inequality (23) and Lemma (3.1).

We recall that the bound (3.1) on the control $\alpha$ is very important for the positivity of the density. As the stability of our optimization scheme is based on the positivity of the variable $m$, the strategy presented in the next section has to include a slope-limiter such that (18)

prevails. Instead of defining $\alpha_{j+1 / 2}^{i}$ through (27), we alternatively denote by $\check{\alpha}_{j+1 / 2}^{i}$ the value obtained in (27) and consider the definition:

$$
\alpha_{j+1 / 2}^{\prime i}=\operatorname{sign}\left(\check{\alpha}_{j+1 / 2}^{i}\right) \cdot \min \left(\lambda,\left|\check{\alpha}_{j+1 / 2}^{i}\right|\right) .
$$

It is easy to check that this modification does not spoil the monotonicity of our procedure, as soon as $\alpha$ satisfies (18).

\subsubsection{The algorithm}

We can now define precisely our optimization algorithm. Suppose that $\alpha^{k}$ is given. The computation of $\alpha^{k+1}$ is achieved as follows.

- Define $v^{k}$ by (19) with $\alpha=\alpha^{k}$.

- Define $m^{0}=m_{0}$ and compute iteratively $m^{i}$ from $m^{i-1}$ according to the sub-steps:

- define $\left(\alpha^{k+1}\right)^{i-1}$ by (28) where $\check{\alpha}_{j+1 / 2}^{i}$ is computed with $v=v^{k}$,

- define $\left(m^{k+1}\right)^{i}$ by (16) with $\alpha^{i-1}=\left(\alpha^{k+1}\right)^{i}$.

A possible termination criterion is obtained by checking the discrete optimality conditions, i.e., given a tolerance threshold Tol $>0$ :

$$
\sup _{1 \leq i \leq N-1,1 \leq j \leq M-1}\left|\frac{\left(m^{k}\right)_{j}^{i}+\left(m^{k}\right)_{j+1}^{i}}{2}\left(\alpha^{k}\right)_{j+1 / 2}^{i}+\left(m^{k}\right)_{j+1 / 2}^{i} \frac{\left(v^{k}\right)_{j+1}^{i+1}-\left(v^{k}\right)_{j}^{i+1}}{d x}\right| \leq \text { Tol. }
$$

The choice of a monotonic algorithm comes from specific properties of the MFG model such as the bi-linearity of the state evolution and special concavity in the cost functional (cf. work in Ref. [18] for a detailed description of the required properties). Let us mention that the algorithm converges numerically very quickly.

\section{Simulations, some results for our model}

We present in this section some numerical results.

First, let us write the particular forms that we have chosen for the state costs. We recall that the heating cost is: $f(t, x)=p(t)(1-\beta x)$. From now on, we take $\beta=0.8$ which means that it is five times cheaper to heat a home that is fully insulated (than a non-insulated one). The choice that we did for the constants in the insulation maintenance cost lead to take: $g(t, x, m)=\frac{x}{0.1+m(t, x)}$. In our algorithm, we choose $\theta=1$. We present in what follows three results that exhibit three qualitatively different phenomena.

In the first one, the unit price of energy $p(t)$ is a constant parameter. With this example, we want to check some simple behaviors of agents. In the second example we look at a non-constant unit price of electricity; more precisely, $p(t)$ reaches a peak. We exhibit in this 
simulation two numerical equilibria. Finally, in the third example, we consider a more realistic case with irreversibility in the investment in insulation material. In the following and for each example, we fixed the horizon $T=1$ and the noise $\frac{\sigma^{2}}{2}=0.07$.

In figures, the horizontal axis is the level of insulation, the depth is the time and the vertical line is the density of agents.

\subsection{Positive externality: the households choose the same technology}

We consider here a Gaussian distribution centered in $x=0.5$ as initial density of players. In other words, the households started already to acquire insulation material, but there are still new technologies that enable them to insulate better the houses.

In the next three figures, we present the curve of the density for three different values of the unit price of energy $p(t)$. The energy will be successively free $(p(t)=0)$, then it will have an intermediate price $(p(t)=3.2)$, and finally it will be expensive $(p(t)=10)$.

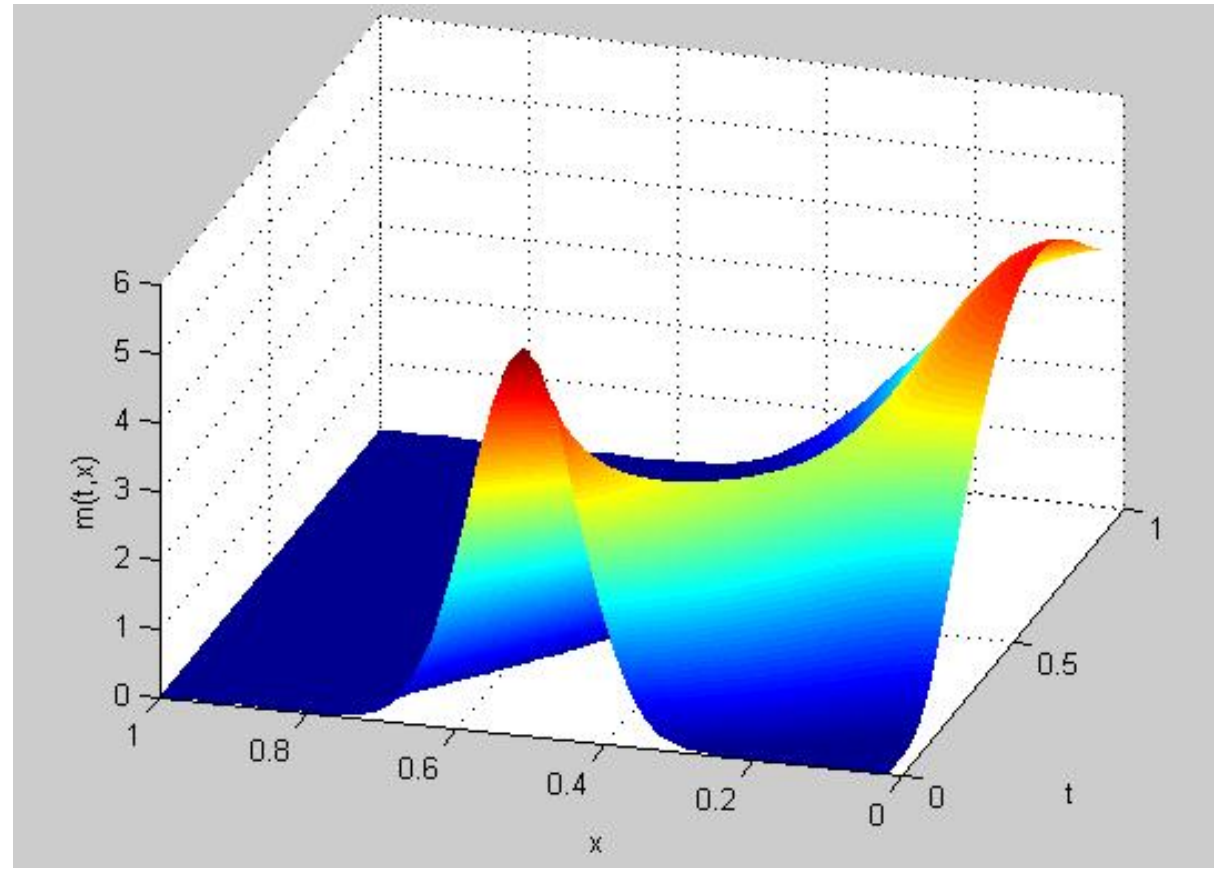

Figure 1: Evolution of $m$ for $p(t)=0$.

On Fig.1, we note that, as expected for very low prices of energy, all agents choose to heat their home and they move to this choice together. On Fig.2, for an intermediate price of energy, the households stay more or less in the same configuration. The important point here is that they are grouped around the same state. And in the third case, we can see on Fig.3 that when the cost related to energy is high, the population wants to be better insulated. And once again, all the players have the same behavior. These solution involve pure bundling.

The economic reasons that explain the behavior observed in the three cases, which consists in bundling (without dispersion), are the positive externality and the economy of scale. These two aspects make the households choose the same technology, the same level of insulation. 


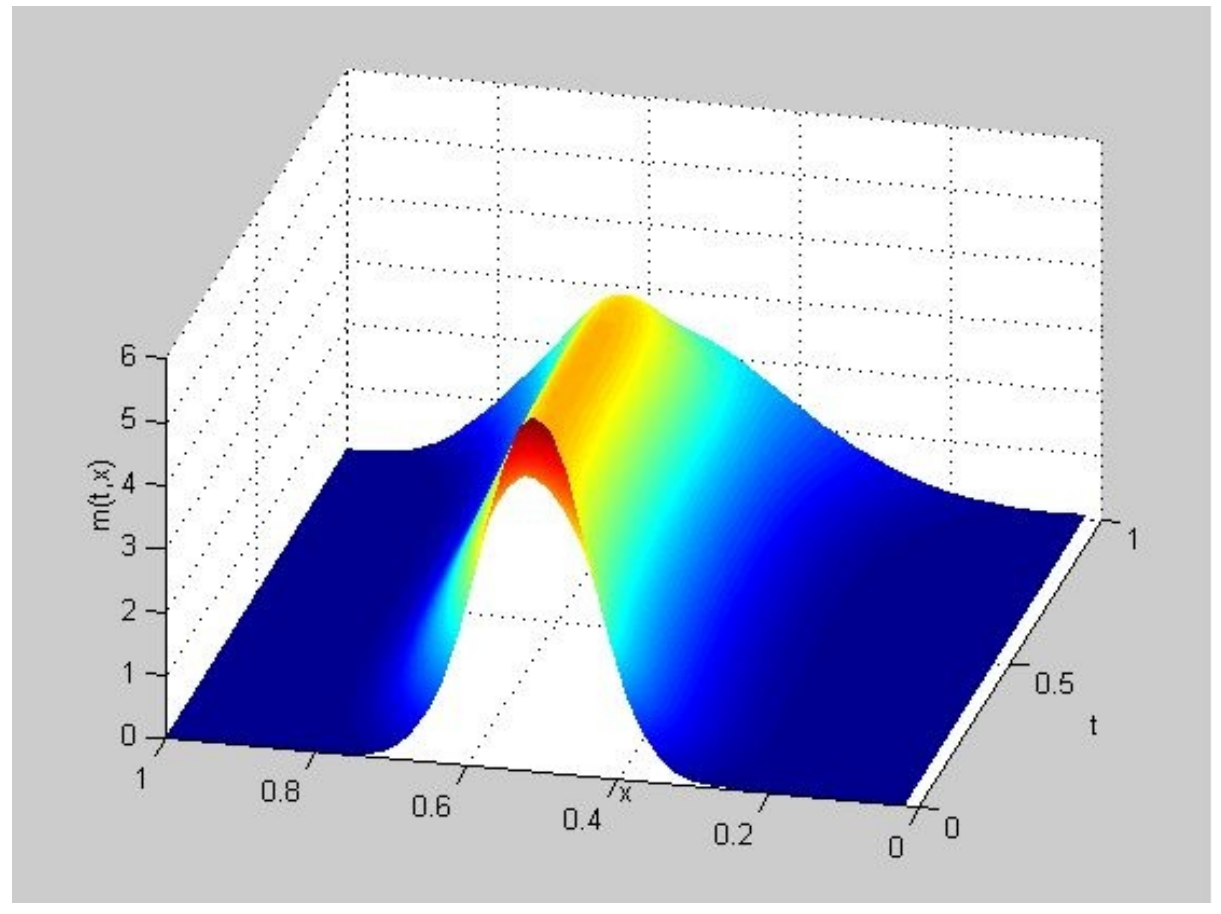

Figure 2: Evolution of $m$ for $p(t)=3.2$.

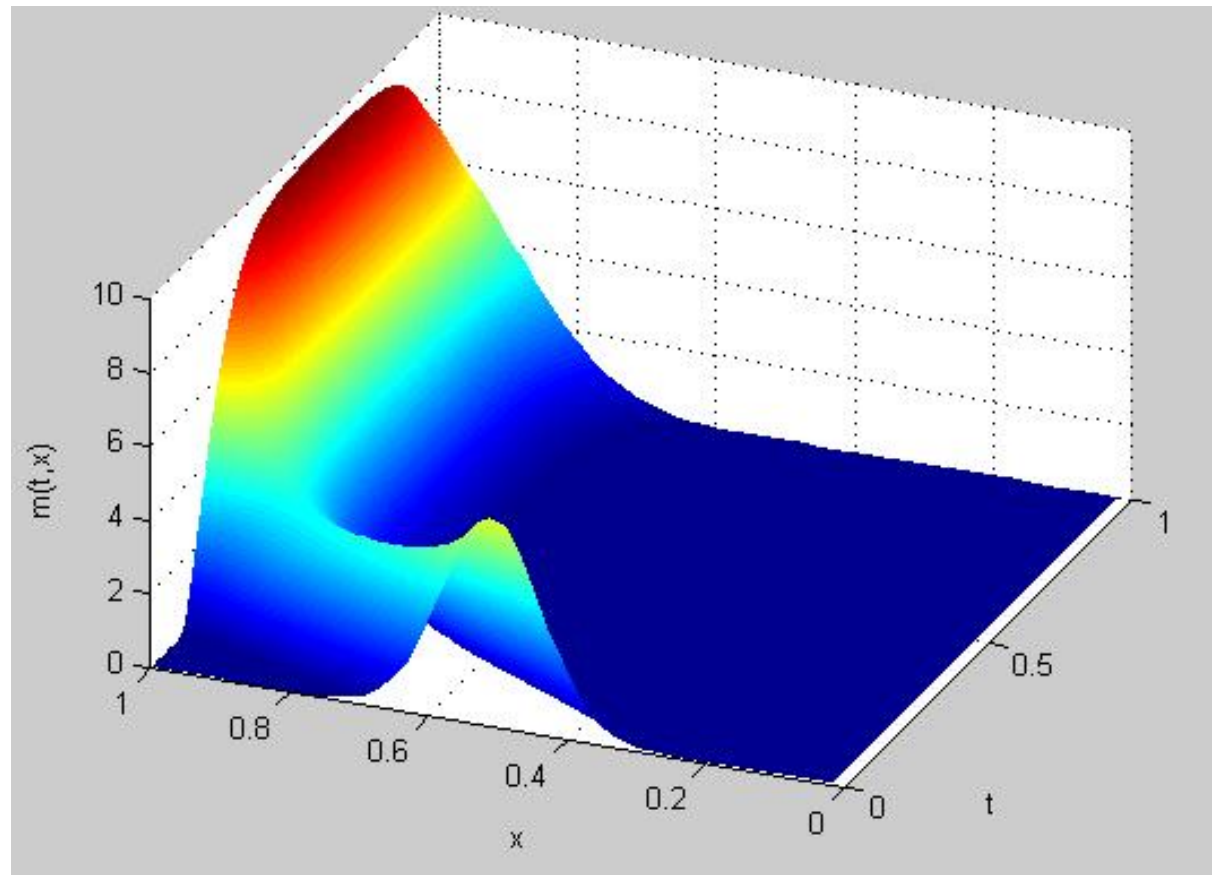

Figure 3: Evolution of $m$ for $p(t)=10$. 


\subsection{Multiplicity of solutions: the possibility of a technological transition}

First, we look at a situation in which the agents are poorly equipped with insulation material at time $t=0$. Thus, the initial density is an approximation of a Dirac mass in $x=0.1$, piecewise linear on $[0,0.1),[0.1,0.3)$, and $[0.3,1)$. Another difference with the first example is the form of the unit price of energy $p(t)$. In the present case, it starts from a low level, then reaches a peak and decreases until its initial level as represented in Fig.4.

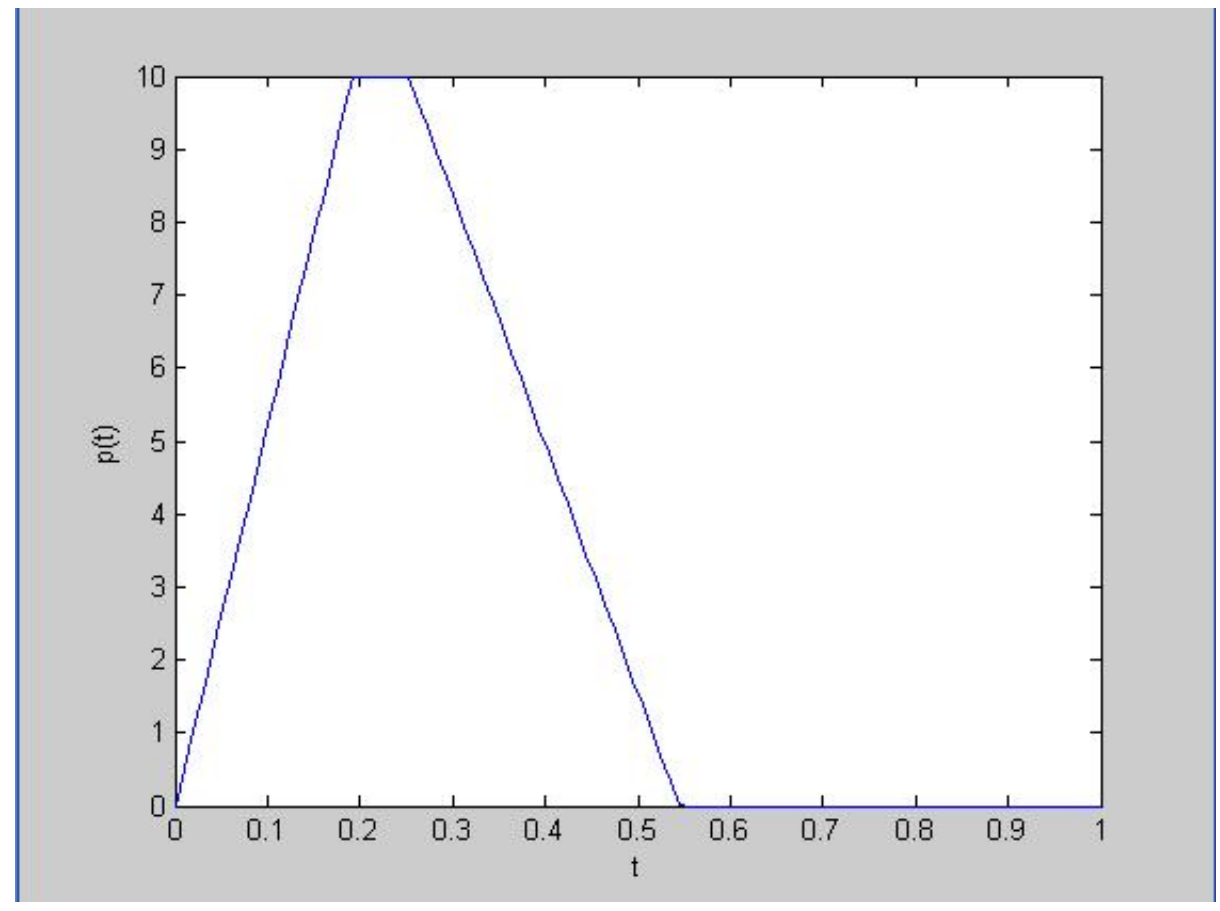

Figure 4: Evolution of the unit price of energy $p(t)$.

With this data, we found numerically two MFG equilibria by taking different initializations for the control variable $\alpha^{0}$. These two numerical solutions are presented in Fig.5 and Fig.6.

Fig. 5 shows the solution corresponding to the choice of high insulation levels. In this case the optimum corresponds to an insulation equilibrium.

The solution in which the households stay heating their apartment stands for a sort of energy consumption equilibrium and is described on Fig.6.

We can explain intuitively the behavior in the following way. If one expects that everybody will still heat their apartment then it is in her interest to do the same, and we get the energy consumption equilibrium. However, if one expects that all the households will improve their insulation, then it is better for her to choose a higher insulation level. In this case we obtain an insulation equilibrium.

By adding a constraint, one can select an equilibrium (e.g. an ecologic equilibrium). In brief, this simple model (based on the MFG) and these simulations (using a monotonic algorithm), enable us to study quantitatively the way the technologies can impact the culture. With this example, we can imagine the design of incentives or policies, in order to change the habits of a population. 


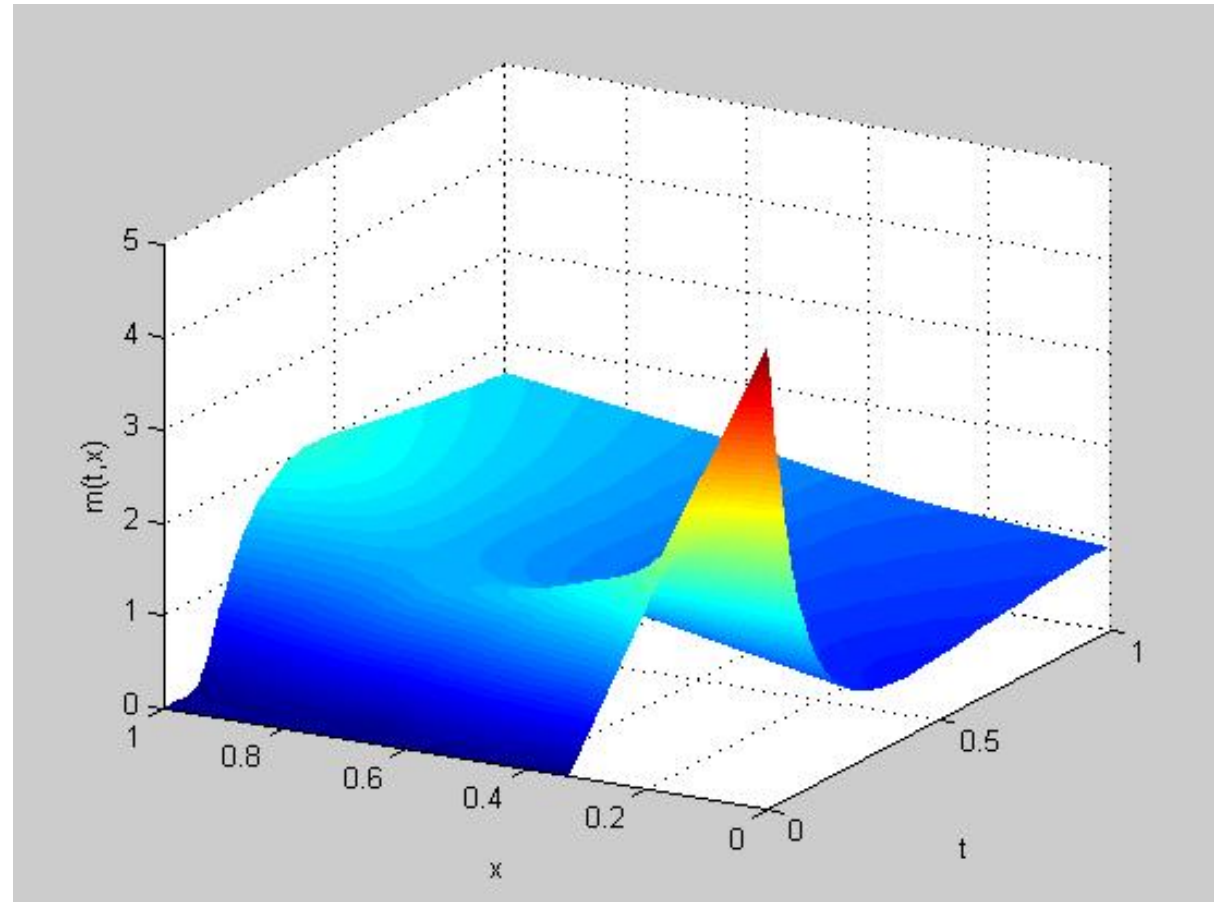

Figure 5: Evolution of $m$, the "insulation equilibrium".

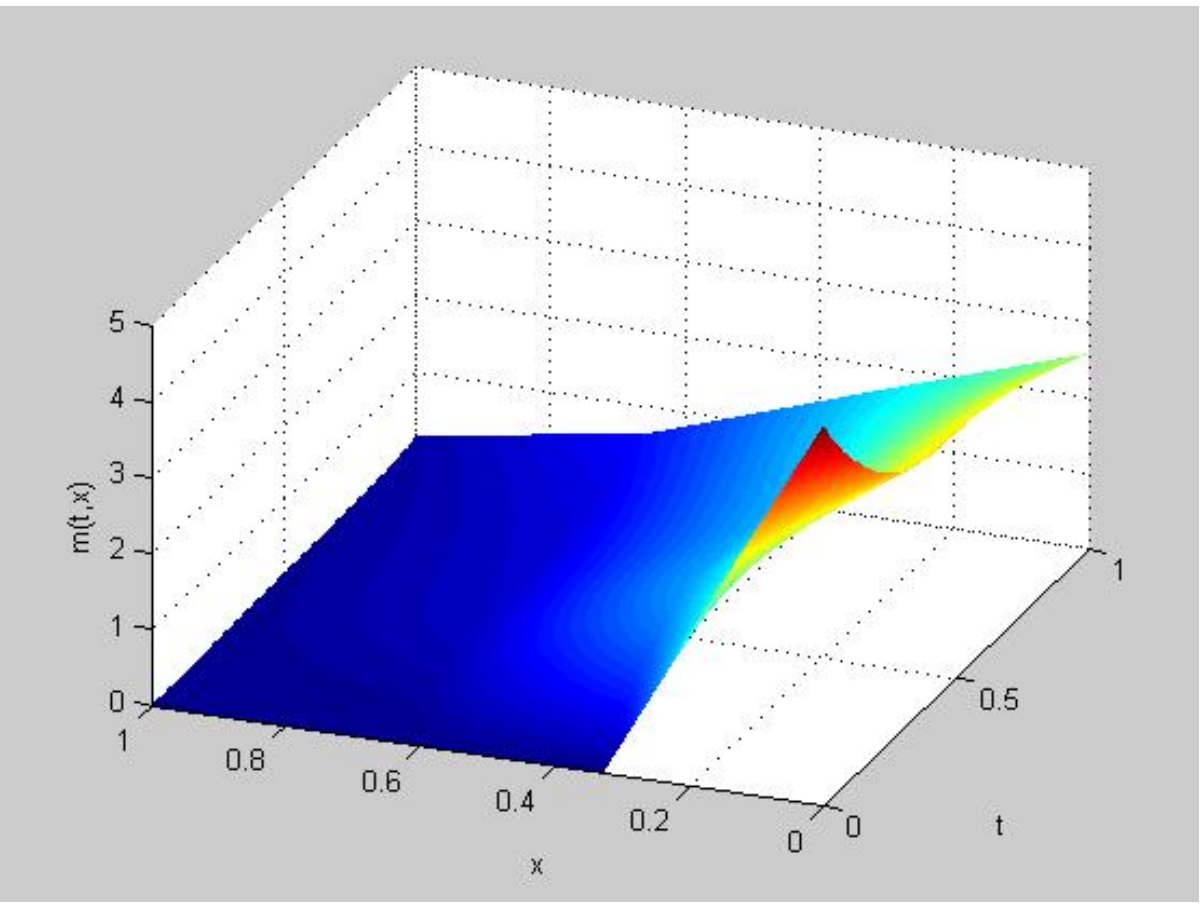

Figure 6: Evolution of $m$, the "energy consumption equilibrium". 


\subsection{Dispersion in the case of irreversibility}

Let us show in a last example how additional constraint on the control can be introduced. We impose $\alpha \geq 0$, so that an investment in insulation material is irreversible. Whereas it is possible to decide to insulate better the apartment, it is not allowed to choose a lower level of insulation. The introduction of such a constraint enables us to handle a more realistic situation. In order to make sense, we have chosen as initial distribution a Gaussian centered in 0.25 - the households did not yet choose a high insulation level. Every other parameter is defined as in the second example (unit price of energy reaching a peak, noise, horizon...).

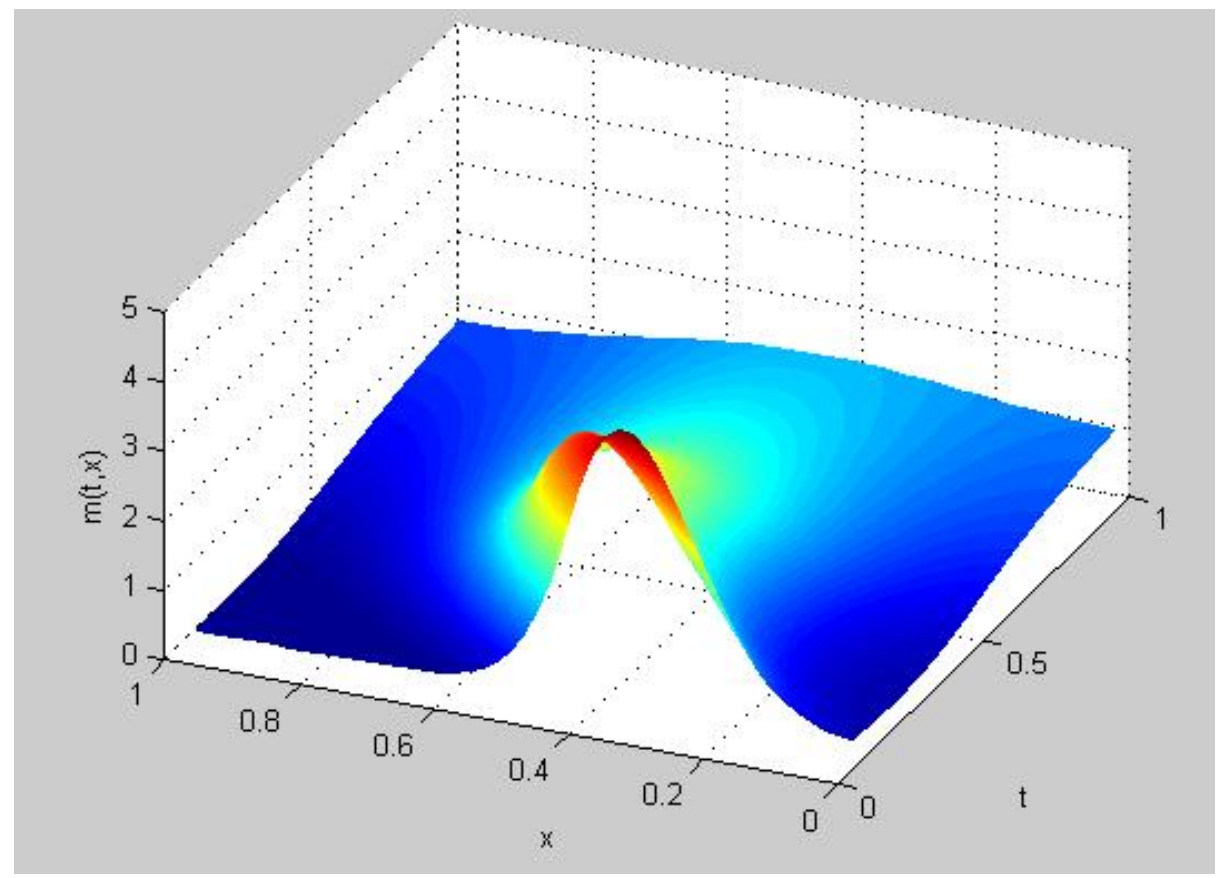

Figure 7: Evolution of $m$, irreversibility and dispersion.

We observe on Fig.7 a dispersion in the household choices. During the first part of the period, the agents choose higher insulation levels, and then there is a spreading. Actually some agents that have chosen to be well equipped with insulation material can not go back. Moreover, in this case, the risk has a more important influence. These two reasons explain intuitively the dispersion that is observed in this example.

\section{Acknowledgments}

This paper was written in the framework of the OTARIE ANR program "ANR-07-BLAN0235 OTARIE" and the Chaire Finance et Développement Durable (supported by EDF and Calyon) after stimulating conversations. It is our pleasure to thank Jean-Michel Lasry for very helpful discussions on the topic, René Aïd for his active interest in the modeling of the choice of technologies, Guillaume Carlier and Bruno Nazaret for their collaboration in proving Theorem (2.1). 


\section{References}

[1] J.-D. Benamou and Y. Brenier, A computational fluid mechanics solution to the MongeKantorovich mass transfer problem , Numer. Math., vol. 84 (3), 2000, pp 375-393.

[2] G. Buttazzo, E. Oudet and C. Jimenez, An optimization problem for mass transportation with congested dynamics, preprint available at:

http://cvgmt.sns.it/people/buttazzo/, retrieved september 2008.

[3] L.C. Evans, Partial Differential Equations, Grad. Stud. Math., AMS, 1998.

[4] G. Carlier, J. Salomon, A monotonic algorithm for the optimal control of the FokkerPlanck equation, to appear in Proc. of the 47 th IEEE Conference on Decision and Control, Cancun, Mexico, Dec. 8-11, 2008.

[5] M. Freidlin, Functional integration and partial differential equations, Annales of Mathematics Studies, Princeton University Press, Study 109, 1985.

[6] C. Hirsch, Numerical Computation of Internal and External Flows: Computational Methods for Inviscid and Viscous Flows (1990), John Wiley \& Sons.

[7] P. E. Kloeden and E. Platen, Numerical solution of stochastic differential equations, Applications of Mathematics (New-York), Springer-Verlag, 1214374, 1992.

[8] V.F. Krotov, Solution Algorithms and Optimization of Control Systems. I, II, Tekh. Kibern., no. 5, 1975, pp. 3-15; no. 6, 1975, pp. 3-13.

[9] A. Lachapelle, Jeux à champ moyen, simulation numérique et application à un modèle de choix technologiques, Mémoire de M2 EDP-MAD, 2006.

[10] J.-M. Lasry and P.-L. Lions, Jeux à champ moyen. I. Le cas stationnaire. (French) [Mean field games. I. The stationary case], C. R. Math. Acad. Sci. Paris, vol. 343 (9), 2006, pp. 619-625.

[11] J.-M. Lasry and P.-L. Lions, Jeux à champ moyen. II. Horizon fini et contrôle optimal. (French) [Mean field games. II. Finite horizon and optimal control], C. R. Math. Acad. Sci. Paris , vol. 343 (10), 2006, pp. 679-684.

[12] J.-M. Lasry and P.-L. Lions, Mean field games, Jpn. J. Math., vol. 2 (1), 2007, pp. 229-260.

[13] J.-L. Lions, Optimal control of systems governed by partial differential equations, SpringerVerlag, 1971.

[14] Y. Maday and G. Turinici, New formulations of monotonically convergent quantum control algorithms, J. Chem. Phys., vol. 118 (18), 2003, pp. 8191-8196.

[15] Y. Maday, J. Salomon and G. Turinici, Monotonic time-discretized schemes in quantum control, Num. Math., vol. 103 (2), 2006, pp. 323-338.

[16] W. H. Press, S. A. Teukolsky, W. T. Vetterling, B. P. Flannery. Numerical Recipes: The Art of Scientific Computing, Third Edition (2007), Cambridge University Press. 
[17] W. Rudin, Real and complex analysis, third ed. McGraw-Hill Book Co., New York, 1987.

[18] J. Salomon and G. Turinici, A monotonic method for solving nonlinear optimal control problems, submitted, preprint hal-00335297 : http://hal.archives-ouvertes.fr/hal$00335297 / \mathrm{fr} /$.

[19] D. Tannor, V. Kazakov and V. Orlov, Control of Photochemical Branching: Novel Procedures for Finding Optimal Pulses and Global Upper Bounds in Time Dependent Quantum Molecular Dynamics, Plenum, Broeckhove J. and Lathouwers L. eds., 1992, pp.347-360.

[20] R. Temam, Navier-Stokes equations, North-Holland, Studies in Mathematics and its Applications, 1984.

[21] W. Zhu and H. Rabitz, A rapid monotonically convergen iteration algorithm for quantum optimal control over the expectation value of a positive definite operator, J. Chem. Phys., vol. 109, 1998, pp. 385-391.

[22] R. Dautray and J.L. Lions, Mathematical analysis and numerical methods for science and technology. Vol. 5, Evolution problems. I, Springer-Verlag, Berlin, 1992,

[23] R. Dautray, Méthodes probabilistes pour les équations de la physique, Eyrolles, Paris, 1989. 